\title{
Representing uncertainty in group decision making through the hesitant information set approach
}

\author{
Manish Aggarwal ${ }^{1}$ \\ Indian Institute of Technology Jodhpur India
}

\begin{abstract}
In multi attribute group decision making (MAGDM), different types of uncertainties co-exist on account of hesitancy, vagueness, and incompleteness besides the diversity in evaluations. To cater to this problem of representing such uncertain evaluations in their original form, new datastructures have been proposed. In this regard, the concept of hesitant information set (HIS) [Applied Soft Computing, 2018] is of great significance. To add to its usefulness in the representation of vague evaluations, it is extended to the continuous and interval-valued domains. A number of illustrative examples are included to show their worth in the real world group decision-making. The basic operations for them, along with their properties, are investigated. A real case-study is included.
\end{abstract}

Keywords: Information set; hesitancy; vagueness; confusion; group multi attribute decision making

\section{Introduction}

An important issue in any multi attribute decision making (MADM) is to represent uncertainty that may be on account of vagueness, hesitancy, individualistic perceptions, or randomness. Such types of uncertainty or imperfection in information is almost inevitable in human decision making. This necessitates suitable data structures in order to retain the originality of the information. This problem assumes yet a bigger magnitude in MAGDMf involving multiple experts with different backgrounds and their own imprefections and different confidence degrees in their evaluations. For example

\footnotetext{
${ }^{1}$ E-mail: ma@iitj.ac.in
} 
MAGDM problem of evaluation of vendors is a classic problem characterizing hesitancy, vagueness and at times ambiguous evaluations by different experts.

There are different approaches proposed in the literature in this context. Hesitant fuzzy set (HFS) [22] is a recent structure that has proved to be perhaps the most useful in MAGDM with it finding several applications [26, $27,28,29,30,31,32,33,34,35,36,37]$. Since its appearance, several of its extensions have also appeared in the literature such as hesitant fuzzy linguistic term sets [38], extended hesitant fuzzy linguistic term set [39].

However, HFS is of limited applicability when it comes to representing different forms of coexisting uncertainty in a decision-making problem. To the best of our knowledge, the literature lacks such a structure, and the closest structure is hesitant information set (HIS) [21]. It is distinguished with HFS by its ability to represent the information as perceived by the agent. But HIS too lacks the ability to represent vagueness in the perceived evaluations. The primary concern of this paper is to develop a structure that can represent multiple forms of uncertainty together in MAGDM problems.

Our endeavour in this study is to also portray the real world uncertainty while retaining its original form. That is, the inherent uncertain nature of the information is not altered in its representation. Inspired by the usefulness of the HIS in the real world decision-making, we accomplish this goal by extending HIS to the domain of vagueness. In contrast to any of the extant structure, the proposed structures helps to determine the best choice in the face of uncertainties due to perceptions, divergence, and vaguness.

The rest of the paper is organized as follows. Section 2 discusses the background. Section 3 gives the necessary preliminaries for the paper. The concept of continuous hesitant information set is proposed in Section 4. The next section is dedicated to highlight the usefulness of the proposed structures in MAGDM. Based on CHIS, another generalization of HIS, called as intervalvalued HIS is presented in Section 6. The basic operations for the proposed structures, along with their properties are devised in Section 7. Section 8 gives a real application of CHIS in group multi attrbute decision making. Section 9 concludes the paper.

\section{Background}

The fuzzy set [2] is popular for representing vagueness through a membership function (MF) that maps an information source value to a partial 
membership degree in the interval $[0,1]$. To cater to the agent's hesitancy in assigning the membership grade, the fuzzy set has been extended as intuitionistic fuzzy set (IFS) [3, 4, 5], Type-2 fuzzy set [24, 25], interval-valued fuzzy set (IVFS) [6, 8, 10, 9], interval-valued fuzzy relation [7], vague fuzzy set [11], and neutrosophic fuzzy set $[12,17,18]$. IFS theory is increasingly gaining attention. An element of IFS has hesitancy in addition to the membership and non-membership grades in the case of a fuzzy set.

Besides hesitancy about the membership grade, an expert may also have multiple membership grades in view for the same information source value. To represent such a situation, fuzzy set is extended as hesitant fuzzy set (HFS) in [22]. A HFS gives multiple membership grades for a single information source value. Most of such datastructures rely on the membership grade to represent imprecision. However, a membership grade may be perceived differently by different onlookers.

In this regard, the concept of information set [20] is especially useful. An information set gives a collection of the perceived information source values. An element of the information set is nothing but an entropy value, and is referred to as the information value. The information set is further extended as the hesitant information set (HIS) in [21]. A HIS gives one or more than one information value(s) corresponding to a single information source value. HIS finds a special significance in group MADM, in which each alternative is evaluated by multiple experts. Often, the experts' evaluations are with different perspectives and therefore conflicting. The well-known aggregation operators $[13,14,15,16]$ have been used in the literature in such situations, which give a single representative aggregated value. However, this results in the loss of diversity in the conflicting informations that otherwise could significantly contribute to the decision making. HIS helps to retain the conflicting subjective evaluations.

\section{Preliminaries}

\subsection{Fuzzy set}

Let a fuzzy set [2] corresponding to a vague concept $X$ and defined over a finite non-empty set of domain values $U=\left\{u_{1}, \ldots, u_{n}\right\}$, is denoted by $F_{X}$. MF for the same is denoted by $\mu_{X}$, representing a mapping:

$$
\mu_{X}: U \rightarrow[0,1],
$$


where $\mu_{X}(u)$ gives the membership grade of $u \in U$ in $X$. The fuzzy set $F_{X}$ is represented by:

$$
F_{X}=\left\{\left(\mu_{X}(u) / u\right): u \in U, \mu_{X}(u) \in[0,1]\right\}
$$

\subsection{Information Set}

Let an item $u_{i}$ of $U$ be described by multiple attributes $E$. The information source value of a particular attribute $e \in E$ for $u_{i}$ is denoted by $I_{e}\left(u_{i}\right)$. A collection of these information source values is expressed as:

$$
\mathcal{I}_{e}=\left\{I_{e}\left(u_{i}\right) \mid \forall u_{i} \in U\right\}
$$

The information set formalism considers that an information source value is perceived differently by different agents, and an agent's perception of a particular information source value, say $I_{e}\left(u_{i}\right)$, depends not only on $I_{e}\left(u_{i}\right)$ but also on $\mathcal{I}_{e}$. The perceived information source value is termed as information value, and is determined through Hanman-Anirban entropy's [19] gain function, shown as:

$$
g_{e}\left(u_{i}\right)=e^{-\left(a_{e}\left(I_{e}\left(u_{i}\right)\right)^{3}+b_{e}\left(I_{e}\left(u_{i}\right)\right)^{2}+c_{e}\left(I_{e}\left(u_{i}\right)\right)+d_{e}\right)^{\alpha_{e}}}
$$

where $g_{e}\left(u_{i}\right)$ gives the perceived value corresponding to $I_{e}\left(u_{i}\right)$; and $a_{e}, b_{e}, c_{e}, d_{e}$ and $\alpha_{e}$ are parameters representing the perception model of the agent for $e$.

It is interesting to note that the form in (4) gives a generalized Gaussian function by replacing the parameter values with moments such as mean and standard deviation of $\mathcal{I}_{e}$. More specifically, let $a_{e}=0, b_{e}=0, c_{e}=\frac{1}{\sigma_{X}}, d_{e}=$ $-\frac{m_{e}}{\sigma_{e}}$ (where $m_{e}$ refers to the mean value $\left.\frac{1}{n} \sum_{i} I_{e}\left(u_{i}\right)\right)$, then we obtain $g_{e}(\cdot)$ as:

$$
g_{e}\left(u_{i}\right)=e^{-\left(\frac{I_{e}\left(u_{i}\right)-m_{e}}{\sigma_{e}}\right)^{\alpha_{e}}}
$$

where $\alpha_{e}$ distinguishes the specific perception model of the agent for $e$. Different values of $\alpha_{e}=0.5,1, \ldots, 5$ correspond to different shapes of $g_{e}(\cdot)$ as shown in Fig. 1. A Gaussian function is obtained at $\alpha_{e}=2$.

An information set is comprised of the information values corresponding to different elements of $U$. It is represented as follows:

$$
\mathcal{S}_{e}=\left\{I_{e}\left(u_{i}\right) g_{e}\left(u_{i}\right)\right\}, \forall u_{i} \in U
$$

where $\mathcal{S}_{e}$ denotes an information set. Each element of the same is shown as 


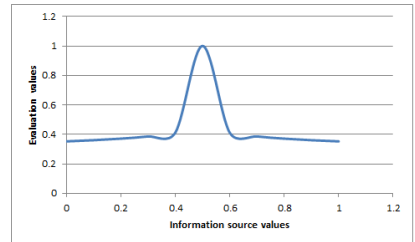

(a) $\alpha=0.1$

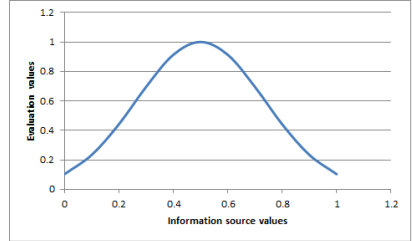

(d) $\alpha=2.0$

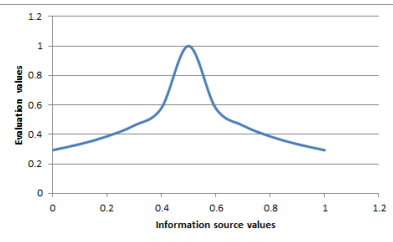

(b) $\alpha=0.5$

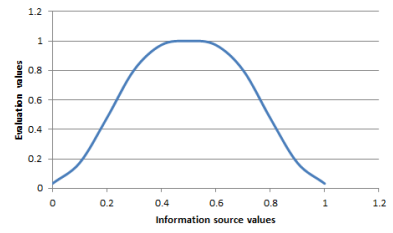

(e) $\alpha=3.0$

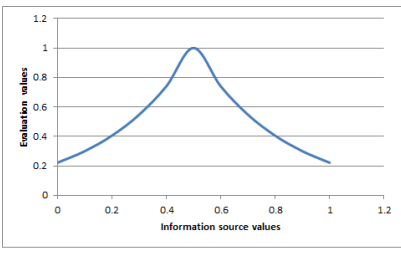

(c) $\alpha=1.0$

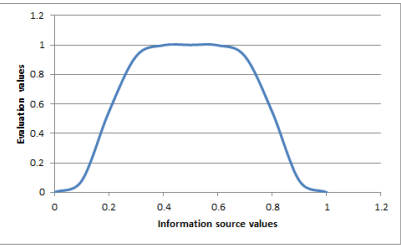

(f) $\alpha=5.0$

Figure 1: The evaluation values of the form $g_{e}\left(u_{i}\right)$, as obtained with information gain function, shown in (5).

follows:

$$
S_{e}\left(u_{i}\right)=I_{e}\left(u_{i}\right) g_{e}\left(u_{i}\right),
$$

The information set formalism thus provides flexibility in representing the different perceptions that different agents may have for an information source value. Besides, the form in (5) also considers the distribution of the information source values, i.e., $\mathcal{I}_{e}$, and hence is context-sensitive. These properties make information set ideal for representing the real world nuances of uncertainty, especially those contributed by the human bias.

\subsection{Hesitant Information Set}

Hesitant information set (HIS) extends information set on the lines of hesitant fuzzy set. HIS refers to a collection of different information values for the same information source value. Let $M=\left\{g_{e}^{(1)}, \ldots, g_{e}^{(N)}\right\}$ denote $N$ different perception models, which an agent may have for $e$. A HIS is defined on $M$ and could be seen as a function $\mathcal{H}: U \rightarrow\{[0,1]\}$. It returns a set of information values lying in the interval $[0,1]$, and is shown as:

$$
\mathcal{H}_{M}(u)=\left\{S_{e}^{(1)}(u), \ldots, S_{e}^{(N)}(u)\right\},
$$


where $S_{e}^{(i)}(u)=I_{e}(u) g_{e}^{(i)}(u)$. HIS $\mathcal{H}_{M}(u)$ thus gives the different perceived information values corresponding to $I_{e}(u)$.

\section{Continuous Hesitant Information Set}

HIS gives a discrete set of crisp information values corresponding to an information source value. Instead, if we consider a continuous interval of information values, emerging from a broad interval of information gains, then what results is a continuous HIS (CHIS).

Let $M=\left[g_{e}^{(1)}, g_{e}^{(N)}\right]$. Then, the corresponding CHIS is denoted by $\tilde{\mathcal{H}}_{M}(u)$, and is shown as:

$$
\begin{aligned}
\tilde{\mathcal{H}}_{M}(u) & =\left[S_{e}^{(1)}(u) \ldots S_{e}^{(N)}(u)\right] \\
& =\left[I_{e}(u) g_{e}^{(1)}(u) \ldots I_{e}(u) g_{e}^{(N)}(u)\right] \\
& =\left[I_{e}(u) g_{e}^{(1)}(u) \ldots I_{e}(u)\left(g_{e}^{(1)}(u)+\pi_{e}(u)\right)\right]
\end{aligned}
$$

where $\pi_{e}(u)=g_{e}^{(N)}(u)-g_{e}^{(1)}(u)$ refers to the width of the information gain interval. The more is this width, the more is the confusion of the agent in crisply associating a single information value to an information source value. CHIS comprises of an interval of information values with $S_{e}^{(1)}(u)$ and $S_{e}^{(N)}(u)$ as this interval's lower and upper bounds.

The information $\Psi_{M}(u)$ associated with $\tilde{\mathcal{H}}_{M}(u)$ is given by

$$
\begin{aligned}
\Psi_{M}(u) & =\int_{i} S_{e}^{(i)}(u) d u \\
& =\int_{i} I_{e}(u) g_{e}^{(i)}(u) d u
\end{aligned}
$$

where $\Psi_{M}(u)$ gives the overall perceived information corresponding to $\tilde{\mathcal{H}}_{e}(u)$.

CHIS allows an expert to give a broad continuous interval as an evaluation of an information source value. For instance, for a subject whose age is unknown, an agent can give the perceived age in terms of a continuous interval. The representation in the form of an interval caters to the imprecision arising due to lack of knowledge of the agent. The choice between HIS and CHIS, as an uncertainty representation structure, depends on the context. CHIS is especially useful in representing imprecise continuous variables, such as time, age, speed etc, where an interval is likely to be more representative of the underlying imprecision than a few discrete values, as in the case of 
HIS. In other instances, such as in a house buying decision, a HIS might be more useful in giving perceived values of the land areas of the houses under consideration.

Besides, the entropic gain function, CHIS can have a fuzzy membership or intuitionistic fuzzy membership as the evaluation of an information source value. In case, when the gain function $g_{e}(u)$ is an intuitionistic fuzzy membership function, i.e. $g_{e}(u)=\left(t_{e}(u), f_{e}(u)\right)$. Then the corresponding CHIS is

$$
\tilde{\mathcal{H}}_{M}(u)=\left[I_{e}(u) t_{e}(u), I_{e}(u)\left(1-f_{e}(u)\right)\right] \text { if } t_{e}(u) \neq 1-f_{e}(u)
$$

where, $M=\left[t_{e}(u), 1-f_{e}(u)\right]$.

Note:- In the sequel, we may omit $M$ at times in the notations for the sake of simplicity.

Proposition 1: Each CHIS is a hesitant information set. Consider $M=$ $\left[g_{e}^{(1)}, g_{e}^{(N)}\right]$. Then the corresponding CHIS is:

$$
\begin{aligned}
\tilde{\mathcal{H}}_{M}(u) & =\left[S_{e}^{(1)}(u), S_{e}^{(N)}(u)\right] \\
& =\mathcal{H}_{M}(u)
\end{aligned}
$$

Since, $\tilde{\mathcal{H}}_{M}(u)$ gives all the possible information values in the given interval, it is also a HIS that is denoted as $\mathcal{H}_{M}(u)$. While a conventional HIS gives a set of possible discrete information values corresponding to an information source value, the proposed CHIS gives a continuous range of the information values.

Proposition 2: For a given CHIS $\tilde{\mathcal{H}}(u)$, the pair of functions $\tilde{\mathcal{H}}^{-}(u)$, $\tilde{\mathcal{H}}^{+}(u)$ give its lower and upper bounds. That is,

$$
\begin{aligned}
\tilde{\mathcal{H}}^{-}(u) & =S_{e}^{(1)}(u) \\
\tilde{\mathcal{H}}^{+}(u) & =S_{e}^{(N)}(u)
\end{aligned}
$$

Proposition 3: Given $\tilde{\mathcal{H}}(u)$, the pair of functions $\tilde{\mathcal{H}}^{-}(u), 1-\tilde{\mathcal{H}}^{+}(u)$ give the constituent elements of a CHIS, denoted by $\tilde{\mathcal{H}}_{\text {env }}(u)$ shown as:

$$
\tilde{\mathcal{H}}_{e n v}(u)=\left[1-\tilde{\mathcal{H}}^{+}(u), \tilde{\mathcal{H}}^{-}(u)\right]
$$

Proposition 4: Let $\tilde{\mathcal{H}}(u), \tilde{\mathcal{H}}_{1}(u)$, and $\tilde{\mathcal{H}}_{2}(u)$ be three different CHISs. Then: 
- $(\overline{\mathcal{H}})_{e n v}(u)=\overline{\tilde{\mathcal{H}}_{e n v}(u)}$

- $\left(\tilde{\mathcal{H}}_{1} \cup \tilde{\mathcal{H}}_{2}\right)_{e n v}(u)=\tilde{\mathcal{H}}_{1, e n v}(u) \cup \tilde{\mathcal{H}}_{2, e n v}(u)$

- $\left(\tilde{\mathcal{H}}_{1} \cap \tilde{\mathcal{H}}_{2}\right)_{e n v}(u)=\tilde{\mathcal{H}}_{1, e n v}(u) \cap \tilde{\mathcal{H}}_{2, e n v}(u)$

\section{Usefulness of the proposed structures in Group Decision Making}

In MAGDM problems, many of the extant structures tend to summarize the diversity in the evaluations gathered through different DMs by way of aggregation operators. However, this does not serve the purpose of group decision making well. On the other hand, some of the extant structures lack a provision to represent the vagueness or imperfection in the evaluations of experts in MAGDM. We illustrate the usefulness of the proposed structures in catering to such situations.

Example 5.1. Let us consider a situation in which a family is deciding upon a car to buy. The family comprises of 3 different members who have their own opinions about the different attributes of the available options. Let there be two attributes:- $e_{1}:$ mileage $(\mathrm{Km} / \mathrm{l})$, and $e_{2}:$ power $(\mathrm{hp})$ describing each of the alternatives.

The proposed structure retains the originality, diversity and vagueness in the evaluations as provided by the experts. Besides, the inherent information set formalism helps to provide the evaluations as perceived by the experts. In this regard, the gain function values provide the perceived values, and the vagueness in the perceived values is represented in the form of an interval.

The corresponding information values are computed through (7). Table 1 shows the normalized information source values, the information gain function intervals, and the corresponding information values, arrived at by applying (9). The last column of Table 1 provides a range of the perceived information values. Each row of Table 1 gives a CHIS; for instance, CHIS corresponding to $u_{6}$ is shown as:

$$
\mathcal{H}\left(u_{6}\right)=[0.5300,0.8100]
$$

Example 5.2. Let us reconsider the problem of the above example. A family member's evaluation of an attribute value is computed by applying (5). The information value of the form $S_{e}(u)$ is computed through (7). The values 
Table 1: Normalized Impact Factors along with the Interval Information Gain Function and Information Values

\begin{tabular}{|l|c|c|c|}
\hline $\mathrm{i}$ & $I_{e}\left(u_{i}\right)$ & {$\left[g_{e}^{(1)}\left(u_{i}\right), g_{e}^{(N)}\left(u_{i}\right)\right]$} & {$\left[S_{e}^{(1)}\left(u_{i}\right), S_{e}^{(N)}\left(u_{i}\right)\right]$} \\
\hline$u_{1}$ & 0.00 & {$[0.0000,0.0000]$} & {$[0.0000,0.0000]$} \\
$u_{2}$ & 0.03 & {$[0.0000,0.0900]$} & {$[0.0000,0.0027]$} \\
$u_{3}$ & 0.12 & {$[0.0100,0.1900]$} & {$[0.0012,0.0228]$} \\
$u_{4}$ & 0.21 & {$[0.1100,0.2400]$} & {$[0.0231,0.0504]$} \\
$u_{5}$ & 0.54 & {$[0.2300,0.5900]$} & {$[0.1242,0.3186]$} \\
$u_{6}$ & 1.00 & {$[0.5300,0.8100]$} & {$[0.5300,0.8100]$} \\
\hline
\end{tabular}

Table 2: Actual Mileage Values with their Evaluations for the Family Members

\begin{tabular}{|c|c|c|c|c|}
\hline $\mathrm{i}$ & $I_{e}\left(u_{i}\right)$ & $g_{e}^{(1)}\left(u_{i}\right)$ & $g_{e}^{(2)}\left(u_{i}\right)$, & $g_{e}^{(3)}\left(u_{i}\right)$ \\
\hline 1 & 0.0944 & 0.4107 & 0.4738 & 0.4000 \\
2 & 0.3259 & 0.8646 & 0.9919 & 0.7901 \\
3 & 0.8828 & 0.3900 & 0.4230 & 0.3844 \\
4 & 0.5951 & 0.9286 & 0.9985 & 0.8677 \\
5 & 0.0000 & 0.2529 & 0.1091 & 0.2809 \\
6 & 1.0000 & 0.2071 & 0.0445 & 0.2452 \\
\hline
\end{tabular}

of $\alpha$ for the family members are given as following: $(2,5,1.5)$ for $e_{1}$, and $(3.5,1.8,2.5)$ for $e_{2}$. Since $\alpha$ is a single value CHIS reduces to yield HIS.

Let $e_{1}$ and $e_{2}$ values for the given choice-set of alternatives are given as $\{6,10.9,22.69,16.60,4.0,25.17\}$ and $\{600,552,188,177,987,53\}$, respectively. These values are normalized as:

$$
I_{e}\left(u_{i}\right)=\frac{I_{e}\left(u_{i}\right)-L}{G-L}
$$

where $G$ and $L$ denote the highest and the smallest values in the set of information source values $\mathcal{I}_{e}$. The normalized information source values along with their evaluations are given in Table 2, and Table 3.

CHIS for $u_{i}$ is determined by applying (8) as:

$$
\mathcal{H}_{M}\left(u_{i}\right)=\left\{I_{e}\left(u_{i}\right) * g_{e}^{(k)}\left(u_{i}\right)\right\}_{k}
$$

where $I_{e}\left(u_{i}\right) * g_{e}^{(k)}\left(u_{i}\right)$ gives the perceived value, corresponding to $I_{e}\left(u_{i}\right)$ of $k^{\text {th }}$ 
Table 3: Actual Power Values with their Evaluations by the Family Members

\begin{tabular}{|c|c|c|c|c|}
\hline $\mathrm{i}$ & $I_{e}\left(u_{i}\right)$ & $g_{e}^{(1)}\left(u_{i}\right)$ & $g_{e}^{(2)}\left(u_{i}\right)$, & $g_{e}^{(3)}\left(u_{i}\right)$ \\
\hline 1 & 0.5856 & 0.9186 & 0.7549 & 0.8422 \\
2 & 0.5342 & 0.9730 & 0.8545 & 0.9263 \\
3 & 0.1445 & 0.7745 & 0.6091 & 0.6857 \\
4 & 0.1327 & 0.7413 & 0.5841 & 0.6554 \\
5 & 1.0000 & 0.0059 & 0.0986 & 0.0403 \\
6 & 0.0000 & 0.2924 & 0.3288 & 0.3138 \\
\hline
\end{tabular}

Table 4: Perceived Mileage Values

\begin{tabular}{|c|c|c|c|c|}
\hline $\mathrm{i}$ & $I_{e}\left(u_{i}\right)$ & $S_{e}^{(1)}\left(u_{i}\right)$ & $S_{e}^{(2)}\left(u_{i}\right)$, & $S_{e}^{(3)}\left(u_{i}\right)$ \\
\hline 1 & 0.0944 & 0.0387 & 0.0447 & 0.0377 \\
2 & 0.3259 & 0.2817 & 0.3232 & 0.2574 \\
3 & 0.8828 & 0.3442 & 0.3734 & 0.3393 \\
4 & 0.5951 & 0.5526 & 0.5942 & 0.5163 \\
5 & 0.0000 & 0.0000 & 0.0000 & 0.0000 \\
6 & 1.0000 & 0.2071 & 0.0445 & 0.2452 \\
\hline
\end{tabular}

family member, in accordance with (6). The perceived (information) values for Tables 2 and 3 are populated in Tables 4 and 5, each row of which gives a CHIS. For example, CHIS $\mathcal{H}_{M}\left(u_{1}\right)=\{0.0387,0.0447,0.0377\}$ emerges from the Ist row of Table 2. Similarly, each CHIS gives the evaluation of a particular attribute value from different perspectives. The best alternative can be determined through the various MCDM models. Aggregation of CHIS can be performed by adding the corresponding values in Tables 4 and 5. Based on the CHISs, so obtained, a consensus can be arrived jointly by the different decision-makers, i.e. the given family members in the present case.

\section{Interval-valued Hesitant Information Set}

In some situations, agent(s) consider(s) multiple information value intervals instead of the crisp information values. To represent such situations, we generalize CHIS as interval-valued hesitant information set (IVHIS), such that each element of IVHIS is an interval of information values. In contrast, there is only a single interval in CHIS and multiple crisp information values 
Table 5: Perceived Power Values

\begin{tabular}{|c|c|c|c|c|}
\hline $\mathrm{i}$ & $I_{e}\left(u_{i}\right)$ & $S_{e}^{(1)}\left(u_{i}\right)$ & $S_{e}^{(2)}\left(u_{i}\right)$, & $S_{e}^{(3)}\left(u_{i}\right)$ \\
\hline 1 & 0.5856 & 0.5379 & 0.4420 & 0.4931 \\
2 & 0.5342 & 0.5197 & 0.4564 & 0.4948 \\
3 & 0.1445 & 0.1119 & 0.0880 & 0.0990 \\
4 & 0.1327 & 0.0983 & 0.0775 & 0.0869 \\
5 & 1.0000 & 0.0059 & 0.0986 & 0.0403 \\
6 & 0.0000 & 0.0000 & 0.0000 & 0.0000 \\
\hline
\end{tabular}

in HIS.

Let $M=\left\{\left[g_{e}^{(1 l)}, g_{e}^{(1 r)}\right], \ldots,\left[g_{e}^{(N l)}, g_{e}^{(N r)}\right]\right\}$, where ${ }^{i l}$ and ${ }^{i r}$ in the superscripts refer to the left and right bounds of $i^{\text {th }}$ possible information gain interval $g_{e}^{i}$. Then, the corresponding IVHIS is denoted by $\mathbb{H}(u)$, and is shown as:

$$
\begin{aligned}
\mathbb{H}(u) & =\left\{\left[S_{e}^{(1 l)}(u), S_{e}^{(1 r)}(u)\right], \ldots,\left[S_{e}^{(N l)}(u), S_{e}^{(N r)}(u)\right]\right\} \\
& =\left\{\left[I_{e}(u) g_{e}^{(1 l)}(u), I_{e}(u) g_{e}^{(1 r)}(u)\right], \ldots,\left[I_{e}(u) g_{e}^{(N l)}(u), I_{e}(u) g_{e}^{(N r)}(u)\right]\right\}
\end{aligned}
$$

It could be seen IVHIS is closely related to CHIS. While, CHIS gives an interval of information values, IVHIS gives multiple such possible intervals. When $g_{e}^{i l}=g_{e}^{i u}$, IVHIS reduces to HIS; and it becomes a CHIS, in case of $\tilde{\mathbb{H}}(u)$ comprising of a single interval.

The net information associated with $\mathbb{H}(u)$ is

$$
\Psi_{M}(u)=\sum_{i} S_{e}^{(i r)}-\sum_{i} S_{e}^{(i l)}
$$

Example 6.1. We reconsider Example 5.1 in group decision making settings in which a DM has an interval-valued gain function, as shown in Table 6. Each of these intervals generates information values in accordance with (9), as shown in Table 7. Each row of Table 7 yields an IVHIS. For instance, the first row shows IVHIS:

$$
\mathbb{H}_{e}\left(u_{1}\right)=\{[0.0000,0.0000],[0.0000,0.0000],[0.0000,0.0000]\}
$$


Table 6: Normalized Impact Factors along with the Interval Information Gain Function Values

\begin{tabular}{|c|c|c|c|c|}
\hline $\mathrm{i}$ & $I_{e}\left(u_{i}\right)$ & {$\left[g_{e}^{(11)}\left(u_{i}\right), g_{e}^{(1 r)}\left(u_{i}\right)\right]$} & {$\left[g_{e}^{(21)}\left(u_{i}\right), g_{e}^{(2 r)}\left(u_{i}\right)\right]$} & {$\left[g_{e}^{(31)}\left(u_{i}\right), g_{e}^{(3 r)}\left(u_{i}\right)\right]$} \\
\hline 1 & 0.00 & {$[0.0000,0.0000]$} & {$[0.0000,0.0000]$} & {$[0.0000,0.0000]$} \\
2 & 0.03 & {$[0.0000,0.0400]$} & {$[0.0210,0.0530]$} & {$[0.0220,0.0900]$} \\
3 & 0.12 & {$[0.0100,0.0500]$} & {$[0.0350,0.1120]$} & {$[0.1011,0.1900]$} \\
4 & 0.21 & {$[0.1100,0.1510]$} & {$[0.1211,0.1804]$} & {$[0.2104,0.2400]$} \\
5 & 0.54 & {$[0.2300,0.2902]$} & {$[0.2442,0.3556]$} & {$[0.2631,0.5900]$} \\
6 & 1.00 & {$[0.5300,0.6200]$} & {$[0.5605,0.6908]$} & {$[0.6231,0.8100]$} \\
\hline
\end{tabular}

Table 7: Interval Information Values

\begin{tabular}{|c|c|c|c|c|}
\hline $\mathrm{i}$ & $I_{e}\left(u_{i}\right)$ & {$\left[S_{e}^{(1 l)}\left(u_{i}\right), S_{e}^{(1 r)}\left(u_{i}\right)\right]$} & {$\left[S_{e}^{(2 l)}\left(u_{i}\right), S_{e}^{(2 r)}\left(u_{i}\right)\right]$} & {$\left[S_{e}^{(3 l)}\left(u_{i}\right), S_{e}^{(3 r)}\left(u_{i}\right)\right]$} \\
\hline 1 & 0.00 & {$[0.0000,0.0000]$} & {$[0.0000,0.0000]$} & {$[0.0000,0.0000]$} \\
2 & 0.03 & {$[0.0000,0.0012]$} & {$[0.0006,0.0016]$} & {$[0.0006,0.0027]$} \\
3 & 0.12 & {$[0.0012,0.0060]$} & {$[0.0025,0.0134]$} & {$[0.0121,0.0228]$} \\
4 & 0.21 & {$[0.0231,0.0317]$} & {$[0.0254,0.0378]$} & {$[0.0441,0.0504]$} \\
5 & 0.54 & {$[0.1242,0.1567]$} & {$[0.1318,0.1920]$} & {$[0.1420,0.3186]$} \\
6 & 1.00 & {$[0.5300,0.6200]$} & {$[0.5605,0.6908]$} & {$[0.6231,0.8100]$} \\
\hline
\end{tabular}


The net information associated with $\mathbb{H}\left(u_{1}\right)$ is

$$
\begin{aligned}
\Psi_{M}\left(u_{1}\right) & =\sum_{i} S_{e}^{(i r)}-\sum S_{e}^{(i l)} \\
& =0.00
\end{aligned}
$$

The above examples demonstrate the abilities of the proposed extensions of HIS in the MAGDM, in which the evaluations for a criterion are both vague and often diversing by different experts. In such applications, CHIS or IVHIS is useful as a datastructure for a holistic representation of such vague evaluations in terms of the information values of different DMs. It also well retains the conflicts in the vague evaluations, as in the reality. We shall also see a detailed application of CHIS in group MADM in Section 8.

\section{Basic Operations}

In this section, we present a few basic operations for the proposed CHIS and IVHIS. Since IVHIS can be seen as a collection of CHISs, we demonstrate the operations on CHISs for the ease of presentation.

\subsection{Extension Principle}

An extension principle is proposed for extending the conventional operations to the proposed CHISs. We consider an operator $\mathbb{O}$ and develop a general extension principle to extend $\mathbb{O}$ on a set of CHISs.

Let $\mathbb{O}$ be a function $\mathbb{O}:[0,1]^{n} \rightarrow[0,1]$. Let $\tilde{\mathscr{H}}$ denote a set of $n$ CHISs $\tilde{\mathscr{H}}=\left\{\tilde{\mathcal{H}}_{1}(u), \ldots, \tilde{\mathcal{H}}_{n}(u)\right\}$, then, the extension of $\mathbb{O}$ on $\tilde{\mathscr{H}}$ is given by:

$$
\mathbb{O}_{\tilde{\mathscr{H}}}=\left\{\mathbb{O}\left(\tilde{\mathcal{H}}_{i}(u)\right)\right\}_{i}
$$

where $\tilde{\mathcal{H}}_{i}(u)=\left[S_{i}^{(1)}(u), S_{i}^{(N)}(u)\right]$

Example 7.1. Let $\tilde{\mathcal{H}}_{1}(u)=[0.4,0.6], \mathcal{H}_{2}(u)=[0.1,0.4]$, and $\mathcal{H}_{3}(u)=$ $[0.5,0.8]$ be the CHISs in $\tilde{\mathscr{H}}=\left\{\tilde{\mathcal{H}}_{1}(u), \tilde{\mathcal{H}}_{2}(u), \tilde{\mathcal{H}}_{3}(u)\right\}$ with $n=3$. If $\mathbb{O}$ is an arithmetic mean operator, say $\cap$, then the result for this operation on $\tilde{\mathcal{H}}_{1}(u), \tilde{\mathcal{H}}_{2}(u)$, and $\tilde{\mathcal{H}}_{3}(u)$ can be obtained by extension of $\cap$ on $\tilde{\mathscr{H}}$, shown as

$$
\begin{aligned}
\Pi_{\tilde{\mathscr{H}}} & =\cap\left\{\tilde{\mathcal{H}}_{1}(u), \tilde{\mathcal{H}}_{2}(u), \tilde{\mathcal{H}}_{3}(u)\right\} \\
& =[\max \{0.4,0.1,0.5\}, \min \{0.6,0.4,0.8\}]
\end{aligned}
$$


The proposed extension principle is remarkably useful in aggregating information in MADM. Let there be $n$ attributes, denoted as $E=\left\{e_{1}, \ldots, e_{n}\right\}$. The information gain for an attribute $e_{i}$ is given by $M_{i}=\left[g_{e_{i}}^{(1)}, g_{e_{i}}^{(N)}\right]$. The CHIS corresponding to the alternative $u$ and the attribute $e_{i}$ is shown as $\tilde{\mathcal{H}}_{M_{i}}(u)=\left[S_{e_{i}}^{(1)}(u), S_{e_{i}}^{(N)}(u)\right]$, where $S_{e_{i}}^{(1)}(u)=I_{e_{i}}(u) g_{e_{i}}^{(1)}(u)$ denotes the lower bound.

If $(\mathbb{O}$ is an aggregation operator then, it yields an aggregated evaluation of $u$ against $E$, which is also a CHIS. The extension principle can be applied to standard complement, union and intersection operations to yield the corresponding operations for CHISs. In the following section, we give the formal definitions of the standard operations for CHIS.

\subsection{Operations and Properties}

Here, we give a few basic operations for CHISs. Let us consider a CHIS $\tilde{\mathcal{H}}_{M}(u)=\left[S_{e}^{(1)}(u), S_{e}^{(N)}(u)\right]$, with lower and upper bounds as $\tilde{\mathcal{H}}_{M}^{-}(u)$, and $\tilde{\mathcal{H}}_{M}^{+}(u)$. The CHIS comprises of a continuous interval of values between these bounds. We now look at the union and intersection operations for CHISs, which are similar to the standard operations.

Let $\tilde{\mathcal{H}}_{M_{1}}(u)=\left[\tilde{\mathcal{H}}_{M_{1}}^{-}(u), \tilde{\mathcal{H}}_{M_{1}}^{+}(u)\right]$ and $\tilde{\mathcal{H}}_{M_{2}}(u)=\left[\tilde{\mathcal{H}}_{M_{2}}^{-}(u), \tilde{\mathcal{H}}_{M_{2}}^{+}(u)\right]$ be two CHISs such that $\left.\tilde{\mathcal{H}}_{M_{1}}^{+}(u)\right]>\tilde{\mathcal{H}}_{M_{2}}^{-}(u)$ or $\tilde{\mathcal{H}}_{M_{2}}^{+}(u)>\tilde{\mathcal{H}}_{M_{1}}^{-}(u)$, then their union is given as:

$$
\tilde{\mathcal{H}}_{M_{1}}(u) \cup \tilde{\mathcal{H}}_{M_{2}}(u)=\left[\min \left\{\tilde{\mathcal{H}}_{M_{1}}^{-}(u), \tilde{\mathcal{H}}_{M_{2}}^{-}(u)\right\}, \max \left\{\tilde{\mathcal{H}}_{M_{1}}^{+}(u), \tilde{\mathcal{H}}_{M_{2}}^{+}(u)\right\}\right]
$$

The intersection of $\tilde{\mathcal{H}}_{M_{1}}(u)$ and $\tilde{\mathcal{H}}_{M_{2}}(u)$ is shown as:

$$
\tilde{\mathcal{H}}_{M_{1}}(u) \cap \tilde{\mathcal{H}}_{M_{2}}(u)=\left[\max \left\{\tilde{\mathcal{H}}_{M_{1}}^{-}(u), \tilde{\mathcal{H}}_{M_{2}}^{-}(u)\right\}, \min \left\{\tilde{\mathcal{H}}_{M_{1}}^{+}(u), \tilde{\mathcal{H}}_{M_{2}}^{+}(u)\right\}\right]
$$

The complement of $\tilde{\mathcal{H}}_{M}(u)$ is defined as:

$$
\overline{\tilde{\mathcal{H}}_{M}}(u)=\overline{\left[\tilde{\mathcal{H}}_{M_{1}}^{-}(u), \tilde{\mathcal{H}}_{M_{1}}^{+}(u)\right]},
$$

where $\overline{\tilde{\mathcal{H}}_{M}}(u)$ gives a set of all the continuous information values outside the interval $\left[\tilde{\mathcal{H}}_{M_{1}}^{-}(u), \tilde{\mathcal{H}}_{M_{1}}^{+}(u)\right]$. 
We adapt the operations + and $\times$ for the proposed CHIS as follows :

$$
\begin{aligned}
\lambda \tilde{\mathcal{H}}_{M}(u) & =\left[\lambda \tilde{\mathcal{H}}_{M_{1}}^{-}(u), \lambda \tilde{\mathcal{H}}_{M_{1}}^{+}(u)\right] \\
\left(\tilde{\mathcal{H}}_{M}\right)^{\lambda}(u) & =\left[\left(\tilde{\mathcal{H}}_{M_{1}}^{-}(u)\right)^{\lambda},\left(\tilde{\mathcal{H}}_{M_{1}}^{+}(u)\right)^{\lambda}\right] \\
\tilde{\mathcal{H}}_{M_{1}}(u)+\tilde{\mathcal{H}}_{M_{2}}(u) & =\int_{\tilde{\mathcal{H}}_{M_{1}}^{-}(u)}^{\tilde{\mathcal{H}}_{M_{1}}^{+}(u)} \mathcal{S}_{1, e}(u) d u+\int_{\tilde{\mathcal{H}}_{M_{2}}^{-}(u)}^{\tilde{\mathcal{H}}_{M_{2}}^{+}(u)} \mathcal{S}_{2, e}(u) d u \\
\tilde{\mathcal{H}}_{M_{1}}(u) \times \tilde{\mathcal{H}}_{M_{2}}(u) & =\int_{\tilde{\mathcal{H}}_{M_{1}}^{-}(u)}^{\tilde{\mathcal{H}}_{M_{1}}^{+}(u)} \mathcal{S}_{1, e}(u) d u \times \int_{\tilde{\mathcal{H}}_{M_{2}}^{-}(u)}^{\tilde{\mathcal{H}}_{M_{2}}^{+}(u)} \mathcal{S}_{2, e}(u) d u
\end{aligned}
$$

where $\mathcal{S}_{1, e}(u)$ and $\mathcal{S}_{2, e}(u)$ in (29) and (30) refer to the continuous informtion values of $\tilde{\mathcal{H}}_{M_{1}}(u)$, and $\tilde{\mathcal{H}}_{M_{2}}(u)$, respectively.

The following basic properties regarding standard union and intersection operations hold true for CHIS.

Proposition 7.1. Let $\mathcal{H}_{M}(u), \mathcal{H}_{M_{1}}(u)$, and $\mathcal{H}_{M_{2}}(u)$ be HISs. Then:

- $\mathcal{H}_{M_{1}}(u) \cup \mathcal{H}_{M_{2}}(u)=\mathcal{H}_{M_{2}}(u) \cup \mathcal{H}_{M_{1}}(u)$

- $\mathcal{H}_{M_{1}}(u) \cap \mathcal{H}_{M_{2}}(u)=\mathcal{H}_{M_{2}}(u) \cap \mathcal{H}_{M_{1}}(u)$

\section{Real Application on Selection of Car}

It is not uncommon to have a difference of opinions among different members of a family, while buying a car. Let us consider a real situation to determine the best car model for a family, while explicitly considering the evaluations of the different family members. In this regard, we shall use the real data for the recent car models [1]. The family comprises of four members, identified as (1), (2), (3) and (4), who evaluate multiple alternatives against a set of desirable attributes :- $\ell$ : length $(\mathrm{mm}), h$ : height $(\mathrm{mm}), \mu$ : power (hp), and $m$ : mileage $(\mathrm{KM} / \mathrm{pl})$.

We normalize the attribute values as :

$$
I_{e}\left(u_{i}\right)=\frac{I_{e}\left(u_{i}\right)-\min \mathcal{I}_{e}}{\max \mathcal{I}_{e}-\min \mathcal{I}_{e}} .
$$

The evaluation behaviour of each of the members is modelled through the $\alpha$ values, as given in Table 8 . For the sake of simplicity, we consider a crisp $\alpha$ value. We apply (5) to evaluate the desirability of each of the family members for a particular attribute value. 
Table 8: Values of $\alpha$ for Family Members

\begin{tabular}{|c|c|c|c|c|}
\hline$k$ & $\alpha_{\ell}^{(k)}$ & $\alpha_{h}^{(k)}$ & $\alpha_{h}^{(k)}$ & $\alpha_{m}^{(k)}$ \\
\hline 1 & 1.8 & 2.9 & 3.6 & 0.6 \\
2 & 0.8 & 1.3 & 2.6 & 4.1 \\
3 & 0.2 & 3.1 & 4.3 & 2.9 \\
4 & 0.7 & 1.4 & 2.1 & 0.9 \\
\hline
\end{tabular}

The subjective utility of an attribute value is computed through (7). The utility values, so determined, for the four family members are populated in Tables .9 and .10. We construct CHISs from the sets of utility values that different family members derive from each of the attribute values, which are shown in Table.11. Since the $\alpha$ values are given to be crisp, CHIS reduces to HIS in the present case. The HISs, corresponding to different attributes of an alternative are aggregated through HFWA operator [23], shown as:

$$
\begin{aligned}
\operatorname{HFWA}\left(\mathcal{H}_{1}, \ldots, \mathcal{H}_{n}\right) & =\oplus_{j=1}^{n}\left(w_{j} h_{j}\right) \\
& =\cup_{\gamma_{1} \in \mathcal{H}_{1}, \ldots, \gamma_{n} \in \mathcal{H}_{n}}\left\{1-\prod_{j=1}^{n}\left(1-\gamma_{j}\right)^{w_{j}}\right\}
\end{aligned}
$$

where $w=\left(w_{1}, w_{2}, \ldots, w_{n}\right)^{T}$ is the weight vector, such that $w_{j} \in[0,1]$, and $\sum_{j=1}^{n} w_{j}=1$.

and are given in Table .12. The score function for each of the CHISs in Table .12 is computed by applying the score function [23]. That is:

$$
s(\mathcal{H})=\frac{1}{\# \mathcal{H}} \sum_{\gamma \in \mathcal{H}} \gamma,
$$

and the values so computed are populated in Table .12. Finally, the given car-models are ranked in accordance with the score values. The rankings, so obtained, are shown in Table 13.

\section{Results and Discussion}

The first column of Table .13 gives the family's likely order of preference for various car models. We note that $\boldsymbol{a}_{58}$ is the most likely model to be 
chosen by the family. CHIS inherits the useful capabilities of both HFS and the information set. HFS permits to consider the diverse evaluations (in the case of MAGDM), or multiple possible evaluations by a single agent, as the case may be. On the other hand, the information set facilitates to model the subjective evaluation behvaviour of a DM. The application demonstrates the usefulness of the proposed extensions in MAGDM by processinbg the vague and divergent information contained in the various HISs.

In practice, the best choice changes with the access of more information, and addition (or omission) of desirable attributes, or alternatives. For instance, in this case-study, the best alternative identified is in the context of choice set $\mathcal{A}=\left\{\boldsymbol{a}_{1}, \ldots, \boldsymbol{a}_{60}\right\}$. It would be easy to think of many more attributes, or alternatives, and the best choice may change with the availability of:

- (a) more information, resulting into change in the desirability curve $g^{X}(\cdot) X=\ell$ or $h$ or $\mu$ or $m$,

- (b) change in the set of desirable attributes, i.e. $\{\ell, h, \mu, m\}$, or

- (c) inclusion (or exclusion) of alternatives in $\mathcal{A}$

The proposed structures help to conveniently cater to such situations. The modelling of subjective evaluation behaviour through $\alpha$ helps to accommodate the changes in an individual's subjective evaluation behaviour. Besides, any changes in $\mathcal{A}$ leads to a different set of information source values $\mathcal{I}_{X}$, and hence a different subjective utility corresponding to an attribute value. Besides, the changes such as the number of attributes, the number of DMs, or the vague evaluation patterns can be easily catered to through the proposed extensions of HIS.

\section{Conclusions and Future Work}

Unlike a hesitant information set, a continuous hesitant information set (CHIS) represents both the vagueness and confusions of the different DMs at the same time. CHIS is further generalized as interval-valued hesitant information set to add to its usefulness in representing vagueness. The proposed extensions of hesitant information sets give useful datastructures for handling multiple vague subjective evaluations of an information source value. In this regard, these structures add to the strength of the heistant information set in representing vague evaluations that are sometimes inevitable in 
a multi attribute decision making. They impart much needed flexibility to the decision-making process by considering divergence in an evaluation of an attribute, vagueness, and confusions of different DMs at the same time.

An extension principle, basic operations, and their properties in the context of the proposed extensions provide useful tools to apply them in any decision-making problem under uncertainty. The proposed extensions emphasize on vagueness and divergence of the evaluations. Their applicability can still be increased by combining them with the rough set theory that deals with incompleteness of information by providing lower and upper approximations. It would also be interesing to conceive their extensions with type-2 fuzzy set, and ignorance functions.

From applications point of view, the proposed structures are of a great significance in reaching consensus, which is still a difficult problem to accomplish in MAGDM. In contrast to the extant consensus reaching methods, the proposed extensions of HIS retain granularity of the linguistic information. With the inherent information set formalism the proposed structures seamlessly and naturally consider the bounded confidence unlike the adjustmentbased approach in the extant structures. In this context, it would be interesting to apply the proposed extensions of HIS in large scale consensus reaching problems such as for social network MAGDM fully considering leadership and bounded confidence in the form of information values. 
Table .9: Utility Values for (1) and (2)

\begin{tabular}{|c|c|c|c|c|c|c|c|c|}
\hline $\mathrm{i}$ & $S_{\ell}^{(1)}\left(u_{i}\right)$ & $S^{(1)}\left(u_{i}\right)$ & $S_{/ 2}^{(1)}\left(u_{i}\right)$ & $S_{m}^{(1)}\left(u_{i}\right)$ & $S_{\ell}^{(2)}\left(u_{i}\right)$ & $S^{(2)}\left(u_{i}\right)$ & $S_{k}^{(2)}\left(u_{i}\right)$ & $S_{m}^{(2)}\left(u_{i}\right)$ \\
\hline 1 & 0.3230 & 0.0100 & 0.0013 & 0.0214 & 0.2926 & 0.0221 & 0.0146 & 0.0000 \\
\hline 2 & 0.5213 & 0.2082 & 0.1301 & 0.3444 & 0.3970 & 0.1565 & 0.1236 & 0.6297 \\
\hline 3 & 0.0671 & 0.1091 & 0.0013 & 0.1102 & 0.1990 & 0.1748 & 0.0146 & 0.1463 \\
\hline 4 & 0.5253 & 0.2186 & 0.1324 & 0.3406 & 0.4824 & 0.1650 & 0.1261 & 0.6271 \\
\hline 5 & 0.1192 & 0.0952 & 0.0130 & 0.1102 & 0.2230 & 0.0809 & 0.0412 & 0.1463 \\
\hline 6 & 0.5304 & 0.1935 & 0.1703 & 0.4325 & 0.4149 & 0.1452 & 0.1676 & 0.5153 \\
\hline 7 & 0.0281 & 0.3347 & 0.0678 & 0.0262 & 0.1718 & 0.3039 & 0.0963 & 0.0002 \\
\hline 8 & 0.4130 & 0.2349 & 0.1820 & 0.3084 & 0.3259 & 0.1791 & 0.1804 & 0.4564 \\
\hline 9 & 0.5340 & 0.0016 & 0.2957 & 0.0214 & 0.4390 & 0.0085 & 0.2629 & 0.0000 \\
\hline 10 & 0.1708 & 0.2839 & 0.1841 & 0.4423 & 0.2422 & 0.2301 & 0.1827 & 0.5178 \\
\hline 11 & 0.2564 & 0.1556 & 0.0002 & 0.0271 & 0.2707 & 0.1185 & 0.0075 & 0.0002 \\
\hline 12 & 0.1942 & 0.2991 & 0.2538 & 0.2593 & 0.2503 & 0.2494 & 0.2526 & 0.4321 \\
\hline 13 & 0.5192 & 0.0000 & 0.0000 & 0.0000 & 0.3941 & 0.0006 & 0.0000 & 0.0000 \\
\hline 14 & 0.0361 & 0.3743 & 0.0334 & 0.2780 & 0.0554 & 0.3742 & 0.0297 & 0.0666 \\
\hline 15 & 0.0902 & 0.3306 & 0.0435 & 0.3397 & 0.0945 & 0.2970 & 0.0386 & 0.6262 \\
\hline 16 & 0.4742 & 0.0000 & 0.2458 & 0.0883 & 0.3562 & 0.0000 & 0.2452 & 0.0770 \\
\hline 17 & 0.0000 & 0.0022 & 0.0000 & 0.2519 & 0.0000 & 0.0844 & 0.0000 & 0.0001 \\
\hline 18 & 0.5274 & 0.0635 & 0.0577 & 0.2083 & 0.4075 & 0.1528 & 0.0515 & 0.3779 \\
\hline 19 & 0.0058 & 0.3981 & 0.0296 & 0.2807 & 0.0194 & 0.3753 & 0.0264 & 0.0906 \\
\hline 20 & 0.4600 & 0.0000 & 0.0015 & 0.0578 & 0.3481 & 0.0005 & 0.0157 & 0.0134 \\
\hline 21 & 0.0000 & 0.0022 & 0.0000 & 0.2519 & 0.0000 & 0.0844 & 0.0000 & 0.0001 \\
\hline 22 & 0.5274 & 0.0635 & 0.0577 & 0.2083 & 0.4075 & 0.1528 & 0.0515 & 0.3779 \\
\hline 23 & 0.0058 & 0.3981 & 0.0296 & 0.2807 & 0.0194 & 0.3753 & 0.0264 & 0.0906 \\
\hline 24 & 0.4600 & 0.0000 & 0.0015 & 0.0578 & 0.3481 & 0.0005 & 0.0157 & 0.0134 \\
\hline 25 & 0.4607 & 0.0407 & 0.0125 & 0.0780 & 0.3485 & 0.0472 & 0.0405 & 0.0493 \\
\hline 26 & 0.4249 & 0.0079 & 0.0000 & 0.0276 & 0.3311 & 0.0195 & 0.0002 & 0.0002 \\
\hline 27 & 0.1886 & 0.1311 & 0.0000 & 0.0000 & 0.2483 & 0.1028 & 0.0035 & 0.0000 \\
\hline 28 & 0.4621 & 0.0001 & 0.0000 & 0.0632 & 0.3492 & 0.0018 & 0.0028 & 0.0204 \\
\hline 29 & 0.0406 & 0.3306 & 0.1495 & 0.1161 & 0.0593 & 0.2970 & 0.1447 & 0.1655 \\
\hline 30 & 0.2360 & 0.3565 & 0.1347 & 0.3940 & 0.1828 & 0.3434 & 0.1285 & 0.5039 \\
\hline 31 & 0.2360 & 0.3991 & 0.0716 & 0.3421 & 0.1828 & 0.3750 & 0.0644 & 0.6284 \\
\hline 32 & 0.4419 & 0.3427 & 0.0681 & 0.2723 & 0.3389 & 0.3180 & 0.0611 & 0.4423 \\
\hline 33 & 0.2231 & 0.0005 & 0.1277 & 0.1297 & 0.2598 & 0.0686 & 0.1210 & 0.2088 \\
\hline 34 & 0.2387 & 0.0000 & 0.0577 & 0.5111 & 0.1845 & 0.0176 & 0.0515 & 0.5317 \\
\hline 35 & 0.2415 & 0.3801 & 0.0658 & 0.4065 & 0.1863 & 0.3764 & 0.0589 & 0.5787 \\
\hline 36 & 0.2452 & 0.2003 & 0.0799 & 0.3083 & 0.1886 & 0.2104 & 0.0723 & 0.4550 \\
\hline 37 & 0.1993 & 0.0053 & 0.1495 & 0.1629 & 0.2519 & 0.0963 & 0.1447 & 0.2973 \\
\hline 38 & 0.1518 & 0.3801 & 0.0658 & 0.4065 & 0.1321 & 0.3764 & 0.0589 & 0.5787 \\
\hline 39 & 0.2846 & 0.1556 & 0.2771 & 0.1820 & 0.2799 & 0.1185 & 0.2462 & 0.3362 \\
\hline 40 & 0.1518 & 0.3801 & 0.0799 & 0.2743 & 0.1321 & 0.3764 & 0.0723 & 0.0402 \\
\hline 41 & 0.1708 & 0.2991 & 0.2069 & 0.3033 & 0.2422 & 0.2494 & 0.2067 & 0.3938 \\
\hline 42 & 0.2369 & 0.3565 & 0.0799 & 0.2746 & 0.1834 & 0.3434 & 0.0723 & 0.0419 \\
\hline 43 & 0.5213 & 0.2663 & 0.0799 & 0.3190 & 0.3970 & 0.2350 & 0.0723 & 0.5521 \\
\hline 44 & 0.5340 & 0.4256 & 0.0799 & 0.2907 & 0.4352 & 0.3290 & 0.0723 & 0.2147 \\
\hline 45 & 0.5340 & 0.4256 & 0.0799 & 0.2907 & 0.4352 & 0.3290 & 0.0723 & 0.2147 \\
\hline 46 & 0.5261 & 0.3440 & 0.1016 & 0.4244 & 0.4049 & 0.3203 & 0.0937 & 0.5643 \\
\hline 47 & 0.4774 & 0.2693 & 0.1450 & 0.2074 & 0.3581 & 0.2361 & 0.1398 & 0.3766 \\
\hline 48 & 0.2060 & 0.4012 & 0.0787 & 0.2613 & 0.1644 & 0.3743 & 0.0712 & 0.0028 \\
\hline 49 & 0.4982 & 0.3994 & 0.0681 & 0.3270 & 0.4509 & 0.2996 & 0.0611 & 0.5952 \\
\hline 50 & 0.4607 & 0.3007 & 0.1123 & 0.3104 & 0.3485 & 0.2516 & 0.1048 & 0.4775 \\
\hline 51 & 0.2323 & 0.3565 & 0.0681 & 0.3028 & 0.1806 & 0.3434 & 0.0611 & 0.3874 \\
\hline 52 & 0.0112 & 0.3503 & 0.0315 & 0.3315 & 0.0286 & 0.3320 & 0.0280 & 0.6106 \\
\hline 53 & 0.0804 & 0.3743 & 0.0589 & 0.3797 & 0.0882 & 0.3742 & 0.0525 & 0.6058 \\
\hline 54 & 0.0240 & 0.4072 & 0.0446 & 0.356219 & 0.0441 & 0.3718 & 0.0396 & 0.6280 \\
\hline 55 & 0.2415 & 0.4117 & 0.0681 & $0.3300^{1}$ & 0.1863 & 0.3692 & 0.0611 & 0.6062 \\
\hline 56 & 0.3653 & 0.2543 & 0.1883 & 0.1780 & 0.3074 & 0.2304 & 0.1872 & 0.3288 \\
\hline 57 & 0.0459 & 0.3915 & 0.0600 & 0.4070 & 0.0636 & 0.2940 & 0.0535 & 0.5783 \\
\hline 58 & 0.5143 & 0.3386 & 0.1757 & 0.3959 & 0.3880 & 0.2650 & 0.1735 & 0.5887 \\
\hline 59 & 0.5339 & 0.3163 & 0.1064 & 0.4522 & 0.4423 & 0.2740 & 0.0986 & 0.5481 \\
\hline 60 & 0.2415 & 0.3743 & 0.0478 & 0.2885 & 0.1863 & 0.3742 & 0.0424 & 0.1849 \\
\hline
\end{tabular}


Table .10: Utility Values for (3) and (4)

\begin{tabular}{|c|c|c|c|c|c|c|c|c|}
\hline $\mathrm{i}$ & $S_{\ell}^{(3)}\left(u_{i}\right)$ & $S_{h}^{(3)}\left(u_{i}\right)$ & $S_{k}^{(3)}\left(u_{i}\right)$ & $S_{m}^{(3)}\left(u_{i}\right)$ & $S_{\ell}^{(4)}\left(u_{i}\right)$ & $S_{h}^{(4)}\left(u_{i}\right)$ & $S_{f}^{(4)}\left(u_{i}\right)$ & $S_{m}^{(4)}\left(u_{i}\right)$ \\
\hline 1 & 0.2742 & 0.0089 & 0.0001 & 0.0013 & 0.2896 & 0.0213 & 0.0336 & 0.0173 \\
\hline 2 & 0.2672 & 0.2126 & 0.1321 & 0.5826 & 0.3782 & 0.1607 & 0.1174 & 0.3893 \\
\hline 3 & 0.3034 & 0.1017 & 0.0001 & 0.1342 & 0.2156 & 0.1704 & 0.0336 & 0.1134 \\
\hline 4 & 0.3053 & 0.2229 & 0.1343 & 0.5751 & 0.4675 & 0.1695 & 0.1200 & 0.3829 \\
\hline 5 & 0.2940 & 0.0969 & 0.0043 & 0.1342 & 0.2346 & 0.0818 & 0.0638 & 0.1134 \\
\hline 6 & 0.2700 & 0.1979 & 0.1708 & 0.5152 & 0.3951 & 0.1489 & 0.1639 & 0.4789 \\
\hline 7 & 0.3160 & 0.3354 & 0.0507 & 0.0023 & 0.1938 & 0.3090 & 0.1120 & 0.0216 \\
\hline 8 & 0.2686 & 0.2390 & 0.1823 & 0.4087 & 0.3166 & 0.1841 & 0.1777 & 0.3220 \\
\hline 9 & 0.2767 & 0.0012 & 0.3136 & 0.0013 & 0.4191 & 0.0079 & 0.2432 & 0.0173 \\
\hline 10 & 0.2874 & 0.2865 & 0.1843 & 0.5177 & 0.2497 & 0.2361 & 0.1802 & 0.4864 \\
\hline 11 & 0.2793 & 0.1595 & 0.0000 & 0.0026 & 0.2721 & 0.1211 & 0.0226 & 0.0224 \\
\hline 12 & 0.2850 & 0.3011 & 0.2540 & 0.4186 & 0.2560 & 0.2554 & 0.2502 & 0.2997 \\
\hline 13 & 0.2669 & 0.0000 & 0.0000 & 0.0000 & 0.3755 & 0.0005 & 0.0000 & 0.0000 \\
\hline 14 & 0.0680 & 0.3743 & 0.0357 & 0.1243 & 0.0575 & 0.3742 & 0.0276 & 0.2560 \\
\hline 15 & 0.0972 & 0.3314 & 0.0463 & 0.5730 & 0.0950 & 0.3023 & 0.0358 & 0.3814 \\
\hline 16 & 0.2660 & 0.0000 & 0.2458 & 0.0809 & 0.3419 & 0.0000 & 0.2439 & 0.0874 \\
\hline 17 & 0.0000 & 0.0010 & 0.0000 & 0.0089 & 0.0000 & 0.0735 & 0.0000 & 0.1982 \\
\hline 18 & 0.2686 & 0.0552 & 0.0611 & 0.3521 & 0.3880 & 0.1463 & 0.0476 & 0.2364 \\
\hline 19 & 0.0306 & 0.3983 & 0.0316 & 0.1462 & 0.0212 & 0.3797 & 0.0246 & 0.2619 \\
\hline 20 & 0.2665 & 0.0000 & 0.0001 & 0.0254 & 0.3351 & 0.0004 & 0.0351 & 0.0532 \\
\hline 21 & 0.0000 & 0.0010 & 0.0000 & 0.0089 & 0.0000 & 0.0735 & 0.0000 & 0.1982 \\
\hline 22 & 0.2686 & 0.0552 & 0.0611 & 0.3521 & 0.3880 & 0.1463 & 0.0476 & 0.2364 \\
\hline 23 & 0.0306 & 0.3983 & 0.0316 & 0.1462 & 0.0212 & 0.3797 & 0.0246 & 0.2619 \\
\hline 24 & 0.2665 & 0.0000 & 0.0001 & 0.0254 & 0.3351 & 0.0004 & 0.0351 & 0.0532 \\
\hline 25 & 0.2665 & 0.0399 & 0.0041 & 0.0588 & 0.3354 & 0.0468 & 0.0630 & 0.0754 \\
\hline 26 & 0.2680 & 0.0068 & 0.0000 & 0.0027 & 0.3208 & 0.0187 & 0.0032 & 0.0229 \\
\hline 27 & 0.2855 & 0.1343 & 0.0000 & 0.0000 & 0.2545 & 0.1047 & 0.0147 & 0.0000 \\
\hline 28 & 0.2664 & 0.0001 & 0.0000 & 0.0330 & 0.3360 & 0.0016 & 0.0128 & 0.0590 \\
\hline 29 & 0.0712 & 0.3314 & 0.1507 & 0.1494 & 0.0613 & 0.3023 & 0.1394 & 0.1205 \\
\hline 30 & 0.1467 & 0.3566 & 0.1365 & 0.5033 & 0.1770 & 0.3464 & 0.1225 & 0.4460 \\
\hline 31 & 0.1467 & 0.3994 & 0.0751 & 0.5783 & 0.1770 & 0.3796 & 0.0596 & 0.3855 \\
\hline 32 & 0.2672 & 0.3431 & 0.0716 & 0.4314 & 0.3273 & 0.3224 & 0.0565 & 0.3153 \\
\hline 33 & 0.2822 & 0.0002 & 0.1299 & 0.1846 & 0.2636 & 0.0575 & 0.1149 & 0.1373 \\
\hline 34 & 0.1476 & 0.0000 & 0.0611 & 0.5317 & 0.1785 & 0.0111 & 0.0476 & 0.5276 \\
\hline 35 & 0.1484 & 0.3801 & 0.0692 & 0.5753 & 0.1801 & 0.3775 & 0.0545 & 0.4692 \\
\hline 36 & 0.1496 & 0.1990 & 0.0834 & 0.4077 & 0.1823 & 0.2097 & 0.0671 & 0.3217 \\
\hline 37 & 0.2845 & 0.0030 & 0.1507 & 0.2649 & 0.2573 & 0.0857 & 0.1394 & 0.1789 \\
\hline 38 & 0.1201 & 0.3801 & 0.0692 & 0.5753 & 0.1301 & 0.3775 & 0.0545 & 0.4692 \\
\hline 39 & 0.2770 & 0.1595 & 0.2957 & 0.3051 & 0.2794 & 0.1211 & 0.2288 & 0.2031 \\
\hline 40 & 0.1201 & 0.3801 & 0.0834 & 0.0963 & 0.1301 & 0.3775 & 0.0671 & 0.2478 \\
\hline 41 & 0.2874 & 0.3011 & 0.2069 & 0.3634 & 0.2497 & 0.2554 & 0.2062 & 0.3112 \\
\hline 42 & 0.1470 & 0.3566 & 0.0834 & 0.0983 & 0.1775 & 0.3464 & 0.0671 & 0.2484 \\
\hline 43 & 0.2672 & 0.2701 & 0.0834 & 0.4865 & 0.3782 & 0.2370 & 0.0671 & 0.3433 \\
\hline 44 & 0.2754 & 0.4320 & 0.0834 & 0.2403 & 0.4152 & 0.3381 & 0.0671 & 0.2841 \\
\hline 45 & 0.2754 & 0.4320 & 0.0834 & 0.2403 & 0.4152 & 0.3381 & 0.0671 & 0.2841 \\
\hline 46 & 0.2682 & 0.3443 & 0.1047 & 0.5631 & 0.3856 & 0.3247 & 0.0876 & 0.4847 \\
\hline 47 & 0.2660 & 0.2733 & 0.1464 & 0.3506 & 0.3436 & 0.2382 & 0.1342 & 0.2352 \\
\hline 48 & 0.1375 & 0.4016 & 0.0823 & 0.0295 & 0.1600 & 0.3793 & 0.0660 & 0.2187 \\
\hline 49 & 0.2833 & 0.4084 & 0.0716 & 0.5295 & 0.4356 & 0.3074 & 0.0565 & 0.3586 \\
\hline 50 & 0.2665 & 0.3026 & 0.1151 & 0.4247 & 0.3354 & 0.2576 & 0.0986 & 0.3260 \\
\hline 51 & 0.1456 & 0.3566 & 0.0716 & 0.3590 & 0.1749 & 0.3464 & 0.0565 & 0.3102 \\
\hline 52 & 0.0418 & 0.3505 & 0.0336 & 0.5483 & 0.0307 & 0.3356 & 0.0261 & 0.3669 \\
\hline 53 & 0.0929 & 0.3743 & 0.0622 & 0.5933 & 0.0890 & 0.3742 & 0.0485 & 0.4402 \\
\hline 54 & 0.0577 & 0.4078 & 0.0474 & 0.595620 & 0.0463 & 0.3777 & 0.0367 & 0.4080 \\
\hline 55 & 0.1484 & 0.4127 & 0.0716 & $0.5425^{20}$ & 0.1801 & 0.3758 & 0.0565 & 0.3642 \\
\hline 56 & 0.2714 & 0.2573 & 0.1885 & 0.2971 & 0.3014 & 0.2319 & 0.1851 & 0.1981 \\
\hline 57 & 0.0747 & 0.4007 & 0.0634 & 0.5750 & 0.0654 & 0.3013 & 0.0495 & 0.4696 \\
\hline 58 & 0.2664 & 0.3468 & 0.1760 & 0.5829 & 0.3699 & 0.2700 & 0.1702 & 0.4586 \\
\hline 59 & 0.2780 & 0.3176 & 0.1093 & 0.5479 & 0.4224 & 0.2798 & 0.0924 & 0.5037 \\
\hline 60 & 0.1484 & 0.3743 & 0.0508 & 0.2192 & 0.1801 & 0.3742 & 0.0393 & 0.2794 \\
\hline
\end{tabular}


Table .11: Evaluations of the attributes by the Family in terms of information values

\begin{tabular}{|c|c|c|c|c|}
\hline $\mathrm{i}$ & $\mathcal{H}_{\ell}^{(1),(2),(3),(4)}\left(u_{i}\right)$ & $\mathcal{H}_{h}^{(1),(}$ & ,(4) $\left(u_{i}\right)$ & $\mathcal{H}_{m}^{(1),(2),(3),(4)}\left(u_{i}\right)$ \\
\hline 1 & $(0.3230,0.2926,0.2742,0.2896)$ & $(0.0100,0.0221,0.0089,0.0213)$ & $(0.0013,0.0146,0.0001,0.0336)$ & $(0.0214,0.0000,0.0013,0.0173)$ \\
\hline 2 & $(0.5213,0.3970,0.2672,0.3782)$ & $(0.2082,0.1565,0.2126,0.1607)$ & $(0.1301,0.1236,0.1321,0.1174)$ & $(0.3444,0.6297,0.5826,0.3893)$ \\
\hline 3 & $(0.0671,0.1990,0.3034,0.2156)$ & $(0.1091,0.1748,0.1017,0.1704)$ & $(0.0013,0.0146,0.0001,0.0336)$ & $(0.1102,0.1463,0.1342,0.1134)$ \\
\hline 4 & $(0.5253,0.4824,0.3053,0.4675)$ & $(0.2186,0.1650,0.2229,0.1695)$ & $(0.1324,0.1261,0.1343,0.1200)$ & $(0.3406,0.6271,0.5751,0.3829)$ \\
\hline 5 & $(0.1192,0.2230,0.2940,0.2346)$ & $(0.0952,0.0809,0.0969,0.0818)$ & $(0.0130,0.0412,0.0043,0.0638)$ & $(0.1102,0.1463,0.1342,0.1134)$ \\
\hline 6 & $(0.5304,0.4149,0.2700,0.3951)$ & $(0.1935,0.1452,0.1979,0.1489)$ & $(0.1703,0.1676,0.1708,0.1639)$ & $(0.4325,0.5153,0.5152,0.4789)$ \\
\hline 7 & $(0.0281,0.1718,0.3160,0.1938)$ & $(0.3347,0.3039,0.3354,0.3090)$ & $(0.0678,0.0963,0.0507,0.1120)$ & $(0.0262,0.0002,0.0023,0.0216)$ \\
\hline 8 & $(0.4130,0.3259,0.2686,0.3166)$ & $(0.2349,0.1791,0.2390,0.1841)$ & $(0.1820,0.1804,0.1823,0.1777)$ & $(0.3084,0.4564,0.4087,0.3220)$ \\
\hline 9 & $(0.5340,0.4390,0.2767,0.4191)$ & $(0.0016,0.0085,0.0012,0.0079)$ & $(0.2957,0.2629,0.3136,0.2432)$ & $(0.0214,0.0000,0.0013,0.0173)$ \\
\hline 10 & $(0.1708,0.2422,0.2874,0.2497)$ & $(0.2839,0.2301,0.2865,0.2361)$ & $(0.1841,0.1827,0.1843,0.1802)$ & $(0.4423,0.5178,0.5177,0.4864)$ \\
\hline 11 & $(0.2564,0.2707,0.2793,0.2721)$ & $(0.1556,0.1185,0.1595,0.1211)$ & $(0.0002,0.0075,0.0000,0.0226)$ & $(0.0271,0.0002,0.0026,0.0224)$ \\
\hline 12 & $(0.1942,0.2503,0.2850,0.2560)$ & $(0.2991,0.2494,0.3011,0.2554)$ & $(0.2538,0.2526,0.2540,0.2502)$ & $(0.2593,0.4321,0.4186,0.2997)$ \\
\hline 13 & $(0.5192,0.3941,0.2669,0.3755)$ & $(0.0000,0.0006,0.0000,0.0005)$ & $(0.0000,0.0000,0.0000,0.0000)$ & $(0.0000,0.0000,0.0000,0.0000)$ \\
\hline 14 & $(0.0361,0.0554,0.0680,0.0575)$ & $(0.3743,0.3742,0.3743,0.3742)$ & $(0.0334,0.0297,0.0357,0.0276)$ & $(0.2780,0.0666,0.1243,0.2560)$ \\
\hline 15 & $(0.0902,0.0945,0.0972,0.0950)$ & $(0.3306,0.2970,0.3314,0.3023)$ & $(0.0435,0.0386,0.0463,0.0358)$ & $(0.3397,0.6262,0.5730,0.3814)$ \\
\hline 16 & $(0.4742,0.3562,0.2660,0.3419)$ & $(0.0000,0.0000,0.0000,0.0000)$ & $(0.2458,0.2452,0.2458,0.2439)$ & $(0.0883,0.077,0.0809,0.08740)$ \\
\hline 17 & $(0.0000,0.0000,0.0000,0.0000)$ & $(0.0022,0.0844,0.0010,0.0735)$ & $(0.0000,0.0000,0.0000,0.0000)$ & $(0.2519,0.0001,0.0089,0.1982)$ \\
\hline 18 & $(0.5274,0.4075,0.2686,0.3880)$ & $(0.0635,0.1528,0.0552,0.1463)$ & $(0.0577,0.0515,0.0611,0.0476)$ & $(0.2083,0.3779,0.3521,0.2364)$ \\
\hline 19 & $(0.0058,0.0194,0.0306,0.0212)$ & $(0.3981,0.3753,0.3983,0.3797)$ & $(0.0296,0.0264,0.0316,0.0246)$ & $(0.2807,0.0906,0.1462,0.2619)$ \\
\hline 20 & $(0.4600,0.3481,0.2665,0.3351)$ & $(0.0000,0.0005,0.0000,0.0004)$ & $(0.0015,0.0157,0.0001,0.0351)$ & $(0.0578,0.0134,0.0254,0.0532)$ \\
\hline 21 & $(0.0000,0.0000,0.0000,0.0000)$ & $(0.0022,0.0844,0.0010,0.0735)$ & $(0.0000,0.0000,0.0000,0.0000)$ & $(0.2519,0.0001,0.0089,0.1982)$ \\
\hline 22 & $(0.5274,0.4075,0.2686,0.3880)$ & $(0.0635,0.1528,0.0552,0.1463)$ & $(0.0577,0.0515,0.0611,0.0476)$ & $(0.2083,0.3779,0$ \\
\hline 23 & $(0.0058,0.0194,0.0306,0.0212)$ & $(0.3981,0.3753,0.3983,0.3797)$ & $(0.0296,0.0264,0.0316,0.0246)$ & $(0.2807,0.0906$ \\
\hline 24 & $(0.4600,0.3481,0.2665,0$. & $(0.0000,0$. & $(0.0015,0$. & $(0.0578$ \\
\hline 25 & $(0.4607,0.3485,0.2665,0.3354)$ & $2,0.0399,0.0468)$ & $5,0.0041,0.0630)$ & $(0.078,0.0$ \\
\hline 26 & $(0.4249,0.3311,0.2680,0.3208)$ & $(0.0079,0.0195,0.0068,0.0187)$ & $(0.0000,0.0002,0.0000,0.0032)$ & $(0.0276,0.0002,0.0$ \\
\hline 27 & $(0.1886,0.2483,0.2855,0.2545)$ & $(0.1311,0.1028,0.1343,0.1047)$ & $(0.0000,0.0035,0.0000,0.0147)$ & $(0.0000,0.0000,0.0000,0.0000)$ \\
\hline 28 & $(0.4621,0.3492,0.2664,0.3360)$ & $(0.0001,0.0018,0.0001,0.0016)$ & $(0.0000,0.0028,0.0000,0.0128)$ & $(0.0632,0.0204,0.0330,0.0590)$ \\
\hline 29 & $(0.0406,0.0593,0.0712,0.0613)$ & $(0.3306,0.2970,0.3314,0.3023)$ & $(0.1495,0.1447,0.1507,0.1394)$ & $(0.1161,0.1655,0.1494,0.1205)$ \\
\hline 30 & $(0.2360,0.1828,0.1467,0.1770)$ & $(0.3565,0.3434,0.3566,0.3464)$ & $(0.1347,0.1285,0.1365,0.1225)$ & $(0.3940,0.5039,0.5033,0.4460)$ \\
\hline 31 & $(0.2360,0.1828,0.1467,0.1770)$ & $(0.3991,0.3750,0.3994,0.3796)$ & $(0.0716,0.0644,0.0751,0.0596)$ & $(0.3421,0.6284,0.5783,0.3855)$ \\
\hline 32 & $(0.4419,0.3389,0.2672,0.3273)$ & $(0.3427,0.3180,0.3431,0.3224)$ & $(0.0681,0.0611,0.0716,0.0565)$ & $(0.2723,0.4423,0.4314,0.3153)$ \\
\hline 33 & $(0.2231,0.2598,0.2822,0.2636)$ & $(0.0005,0.0686,0.0002,0.0575)$ & $(0.1277,0.1210,0.1299,0.1149)$ & $(0.1297,0.2088,0.1846,0.1373)$ \\
\hline 34 & $(0.2387,0.1845,0.1476,0.1785)$ & $(0.0000,0.0176,0.0000,0.0111)$ & $(0.0577,0.0515,0.0611,0.0476)$ & $(0.5111,0.5317,0.5317,0$. \\
\hline 35 & $(0.2415,0.1$ & $(0.3801$, & $(0.0658,0$. & $(0.4065$ \\
\hline 36 & $6,0.1496,0.1823)$ & $(0.2003,0.2104,0.1990,0.2097)$ & $(0.0799,0.0$ & $(0.3083,0$ \\
\hline 37 & $(0.1993,0.2519,0.2845,0.2573)$ & $(0.0053,0.0963,0.0030,0.0857)$ & $(0.1495,0.1447,0.1507,0.1394)$ & $(0.1629,0.2973,0.2649,0.1789)$ \\
\hline 38 & $(0.1518,0.1321,0.1201,0.1301)$ & $(0.3801,0.3764,0.3801,0.3775)$ & $(0.0658,0.0589,0.0692,0.0545)$ & $(0.4065,0.5787,0.5753,0.4692)$ \\
\hline 39 & $(0.2846,0.2799,0.2770,0.2794)$ & $(0.1556,0.1185,0.1595,0.1211)$ & $(0.2771,0.2462,0.2957,0.2288)$ & $(0.1820,0.3362,0.3051,0.2031)$ \\
\hline 40 & $(0.1518,0.1321,0.1201,0.1301)$ & $(0.3801,0.3764,0.3801,0.3775)$ & $(0.0799,0.0723,0.0834,0.0671)$ & $(0.2743,0.0402,0.0963,0.2478)$ \\
\hline 41 & $(0.1708,0.2422,0.2874,0.2497)$ & $(0.2991,0.2494,0.3011,0.2554)$ & $(0.2069,0.2067,0.2069,0.2062)$ & $(0.3033,0.3938,0.3634,0.3112)$ \\
\hline 42 & $(0.2369,0.1834,0.1470,0.1775)$ & $(0.3565,0.3434,0.3566,0.3464)$ & $(0.0799,0.0723,0.0834,0.0671)$ & $(0.2746,0.0419,0.0983,0.2484)$ \\
\hline 43 & $(0.5213,0.3970,0.2672,0.3782)$ & $(0.2663,0.2350,0.2701,0.2370)$ & $(0.0799,0.0723,0.0834,0.0671)$ & $(0.3190,0.5521,0.4865,0.3433)$ \\
\hline 44 & $(0.5340,0.4352,0.2754,0.4152)$ & $(0.4256,0.3290,0.4320,0.3381)$ & $(0.0799,0.0723,0.0834,0.0671)$ & $(0.2907,0.2147,0.2403,0.2841)$ \\
\hline 45 & $(0.5340,0.4352,0.2754,0.4152)$ & $(0.4256,0.3290,0.4320,0.3381)$ & $(0.0799,0.0723,0.0834,0.0671)$ & $(0.2907,0.2147,0.2403,0.2841)$ \\
\hline 46 & $(0.5261,0.4049,0.2682,0.3856)$ & $(0.3440,0.3203,0.3443,0.3247)$ & $(0.1016,0.0937,0.1047,0.0876)$ & $(0.4244,0.5643,0.5631,0.4847)$ \\
\hline 47 & $(0.4774,0.3581,0.2660,0.3436)$ & $(0.2693,0.2361,0.2733,0.2382)$ & $(0.1450,0.1398,0.1464,0.1342)$ & $(0.2074,0.3766,0.3506,0.2352)$ \\
\hline 48 & $(0.2060,0.1644,0.1375,0.1600)$ & $(0.4012,0.3743,0.4016,0.3793)$ & $(0.0787,0.0712,0.0823,0.0660)$ & $(0.2613,0.0028,0.0295,0.2187)$ \\
\hline 49 & $(0.4982,0.4509,0.2833,0.4356)$ & $(0.3994,0.2996,0.4084,0.3074)$ & $(0.0681,0.0611,0.0716,0.0565)$ & $(0.3270,0.5952,0.5295,0.3586)$ \\
\hline 50 & $(0.4607,0.3485,0.2665,0.3354)$ & $(0.3007,0.2516,0.3026,0.2576)$ & $(0.1123,0.1048,0.1151,0.0986)$ & $(0.3104,0.4775,0.4247,0.3260)$ \\
\hline 51 & $(0.2323,0.1806,0.1456,0.1749)$ & $(0.3565,0.3434,0.3566,0.3464)$ & $(0.0681,0.0611,0.0716,0.0565)$ & $(0.3028,0.3874,0.3590,0.3102)$ \\
\hline 52 & $(0.0112,0.0286,0.0418,0.0307)$ & $(0.3503,0.3320,0.3505,0.3356)$ & $(0.0315,0.0280,0.0336,0.0261)$ & $(0.3315,0.6106,0.5483,0.3669)$ \\
\hline 53 & $(0.0804,0.0882,0.0929,0.0890)$ & $(0.3743,0.372 \pm, 0.3743,0.3742)$ & $(0.0589,0.0525,0.0622,0.0485)$ & $(0.3797,0.6058,0.5933,0.4402)$ \\
\hline 54 & $(0.0240,0.0441,0.0577,0.0463)$ & $(0.4072,0.3718,0.4078,0.3777)$ & $(0.0446,0.0396,0.0474,0.0367)$ & $(0.3562,0.6280,0.5956,0.4080)$ \\
\hline 55 & $(0.2415,0.1863,0.1484,0.1801)$ & $(0.4117,0.3692,0.4127,0.3758)$ & $(0.0681,0.0611,0.0716,0.0565)$ & $(0.3300,0.6062,0.5425,0.3642)$ \\
\hline 56 & $(0.3653,0.3074,0.2714,0.3014)$ & $(0.2543,0.2304,0.2573,0.2319)$ & $(0.1883,0.1872,0.1885,0.1851)$ & $(0.1780,0.3288,0.2971,0.1981)$ \\
\hline 57 & $(0.0459,0.0636,0.0747,0.0654)$ & $(0.3915,0.2940,0.4007,0.3013)$ & $(0.0600,0.0535,0.0634,0.0495)$ & $(0.4070,0.5783,0.5750,0.4696)$ \\
\hline 58 & $(0.5143,0.3880,0.2664,0.3699)$ & $(0.3386,0.2650,0.3468,0.2700)$ & $(0.1757,0.1735,0.1760,0.1702)$ & $(0.3959,0.5887,0.5829,0.4586)$ \\
\hline 59 & $(0.5339,0.4423,0.2780,0.4224)$ & $(0.3163,0.2740,0.3176,0.2798)$ & $(0.1064,0.0986,0.1093,0.0924)$ & $(0.4522,0.5481,0.5479,0.5037)$ \\
\hline 60 & $(0.2415,0.1863,0.1484,0.1801)$ & $(0.3743,0.3742,0.3743,0.3742)$ & $(0.0478,0.0424,0.0508,0.0393)$ & $(0.2885,0.1849,0.2192,0.2794)$ \\
\hline
\end{tabular}




\begin{tabular}{|c|c|c|c|c|}
\hline $\mathrm{i}$ & $\mathcal{H}_{\ell, h, \ell, m}^{(1),(2),(3),(4)}\left(u_{i}\right)$ & $s\left(\mathcal{H}_{\ell, \ell, k, m}^{(1),(2),(3),(4)}\left(u_{i}\right)\right)$ & $a_{i}$ & $\mathcal{H}_{\ell, \ell, /, m}^{(1),(2),(3),(4)}\left(u_{i}\right)$ \\
\hline 1 & $(0.2951,0.0156,0.0125,0.0100)$ & 0.0833 & 58 & 0.3461 \\
\hline 2 & $(0.3978,0.1849,0.1258,0.5012)$ & 0.3024 & 46 & 0.3365 \\
\hline 3 & $(0.2006,0.1397,0.0125,0.1262)$ & 0.1198 & 59 & 0.3349 \\
\hline 4 & $(0.4509,0.1944,0.1282,0.4961)$ & 0.3174 & 49 & 0.3267 \\
\hline 5 & $(0.2202,0.0887,0.0309,0.1262)$ & 0.1165 & 4 & 0.3174 \\
\hline 6 & $(0.4098,0.1717,0.1682,0.4866)$ & 0.3091 & 6 & 0.3091 \\
\hline 7 & $(0.1838,0.3209,0.0820,0.0126)$ & 0.1498 & 2 & 0.3024 \\
\hline 8 & $(0.3331,0.2098,0.1806,0.3769)$ & 0.2751 & 10 & 0.2933 \\
\hline 9 & $(0.4243,0.0048,0.2794,0.0100)$ & 0.1796 & 43 & 0.2899 \\
\hline 10 & $(0.2387,0.2596,0.1828,0.4920)$ & 0.2933 & 35 & 0.2858 \\
\hline 11 & $(0.2697,0.1389,0.0076,0.0131)$ & 0.1073 & 31 & 0.2852 \\
\hline 12 & $(0.2471,0.2766,0.2527,0.3567)$ & 0.2833 & 44 & 0.2847 \\
\hline 13 & $(0.3957,0.0003,0.0000,0.0000)$ & 0.0990 & 45 & 0.2847 \\
\hline 14 & $(0.0543,0.3743,0.0316,0.1860)$ & 0.1616 & 12 & 0.2833 \\
\hline 15 & $(0.0942,0.3155,0.0411,0.4947)$ & 0.2364 & 50 & 0.2829 \\
\hline 16 & $(0.3641,0.0000,0.2452,0.0834)$ & 0.1732 & 30 & 0.2829 \\
\hline 17 & $(0.0000,0.0411,0.0000,0.1219)$ & 0.0408 & 55 & 0.2801 \\
\hline 18 & $(0.4050,0.1056,0.0545,0.2974)$ & 0.2156 & 32 & 0.2781 \\
\hline 19 & $(0.0193,0.3879,0.0281,0.1987)$ & 0.1585 & 8 & 0.2751 \\
\hline 20 & $(0.3563,0.0002,0.0132,0.0376)$ & 0.1018 & 38 & 0.2717 \\
\hline 21 & $(0.0000,0.0411,0.0000,0.1219)$ & 0.0408 & 41 & 0.2665 \\
\hline 22 & $(0.4050,0.1056,0.0545,0.2974)$ & 0.2156 & 47 & 0.2645 \\
\hline 23 & $(0.0193,0.3879,0.0281,0.1987)$ & 0.1585 & 53 & 0.2579 \\
\hline 24 & $(0.3563,0.0002,0.0132,0.0376)$ & 0.1018 & 56 & 0.2491 \\
\hline 25 & $(0.3567,0.0437,0.0303,0.0654)$ & 0.1240 & 54 & 0.2468 \\
\hline 26 & $(0.3387,0.0132,0.0009,0.0134)$ & 0.0916 & 57 & 0.2451 \\
\hline 27 & $(0.2450,0.1183,0.0046,0.0000)$ & 0.0920 & 15 & 0.2364 \\
\hline 28 & $(0.3574,0.0009,0.0039,0.0441)$ & 0.1016 & 39 & 0.2353 \\
\hline 29 & $(0.0582,0.3155,0.1461,0.1381)$ & 0.1645 & 51 & 0.235 \\
\hline 30 & $(0.1863,0.3508,0.1306,0.4637)$ & 0.2829 & 52 & 0.2195 \\
\hline 31 & $(0.1863,0.3884,0.0677,0.4983)$ & 0.2852 & 18 & 0.2156 \\
\hline 32 & $(0.3469,0.3316,0.0643,0.3695)$ & 0.2781 & 22 & 0.2156 \\
\hline 33 & $(0.2575,0.0322,0.1234,0.1657)$ & 0.1447 & 60 & 0.2134 \\
\hline 34 & $(0.1880,0.0072,0.0545,0.5256)$ & 0.1938 & 36 & 0.2123 \\
\hline 35 & $(0.1898,0.3785,0.0621,0.5127)$ & 0.2858 & 42 & 0.1963 \\
\hline 36 & $(0.1922,0.2049,0.0757,0.3762)$ & 0.2123 & 34 & 0.1938 \\
\hline 37 & $(0.2489,0.0486,0.1461,0.2281)$ & 0.1679 & 48 & 0.1917 \\
\hline 38 & $(0.1336,0.3785,0.0621,0.5127)$ & 0.2717 & 40 & 0.1896 \\
\hline 39 & $(0.2802,0.1389,0.2624,0.2595)$ & 0.2353 & 9 & 0.1796 \\
\hline 40 & $(0.1336,0.3785,0.0757,0.1705)$ & 0.1896 & 16 & 0.1732 \\
\hline 41 & $(0.2387,0.2766,0.2067,0.3440)$ & 0.2665 & 37 & 0.1679 \\
\hline 42 & $(0.1869,0.3508,0.0757,0.1716)$ & 0.1963 & 29 & 0.1645 \\
\hline 43 & $(0.3978,0.2523,0.0757,0.4337)$ & 0.2899 & 14 & 0.1616 \\
\hline 44 & $(0.4221,0.3830,0.0757,0.2581)$ & 0.2847 & 19 & 0.1585 \\
\hline 45 & $(0.4221,0.3830,0.0757,0.2581)$ & 0.2847 & 23 & 0.1585 \\
\hline 46 & $(0.4033,0.3334,0.0969,0.5125)$ & 0.3365 & 7 & 0.1498 \\
\hline 47 & $(0.3659,0.2544,0.1414,0.2962)$ & 0.2645 & 33 & 0.1447 \\
\hline 48 & $(0.1673,0.3892,0.0746,0.1355)$ & 0.1917 & 25 & 0.124 \\
\hline 49 & $(0.4222,0.3557,0.0643,0.4645)$ & 0.3267 & 3 & 0.1198 \\
\hline 50 & $(0.3567,0.2785,0.1077,0.3886)$ & 0.2829 & 5 & 0.1165 \\
\hline 51 & $(0.1840,0.3508,0.0643,0.3408)$ & 0.2350 & 11 & 0.1073 \\
\hline 52 & $(0.0281,0.3422,0.0298,0.4777)$ & 0.2195 & 20 & 0.1018 \\
\hline 53 & $(0.0876,0.3743,0.0555,0.5143)$ & 0.2579 & 24 & 0.1018 \\
\hline 54 & $(0.0431,0.3913,0.0421,0.5107)$ & 0.2208 & 28 & 0.1016 \\
\hline 55 & $(0.1898,0.3927,0.0643,0.4737)$ & 0.2801 & 13 & 0.099 \\
\hline 56 & $(0.3122,0.2436,0.1873,0.2532)$ & 0.2491 & 27 & 0.092 \\
\hline 57 & $(0.0625,0.3487,0.0566,0.5127)$ & 0.2451 & 26 & 0.0916 \\
\hline 58 & $(0.3912,0.3061,0.1739,0.5133)$ & 0.3461 & 1 & 0.0833 \\
\hline 59 & $(0.4262,0.2972,0.1017,0.5145)$ & 0.3349 & 17 & 0.0408 \\
\hline 60 & $(0.1898,0.3743,0.0451,0.2442)$ & 0.2134 & 21 & 0.0408 \\
\hline
\end{tabular}




\section{Compliance with Ethical Standards}

- Author declares that there is no conflict of interest.

- This article does not contain any studies with human participants or animals performed by any of the authors.

\section{References}

[1] https://auto.ndtv.com, 2017.

[2] L. A. Zadeh, Fuzzy sets, Information and Control, 8 (3), 1965, pp. 338-353

[3] K. Atanassov, Intuitionistic fuzzy sets. in: V. Sgurev, (Ed.), VII ITKR's Session. Sofia, June 1983, Central Sci. and Techn. Library, Bulg. Academy of Sciences, 1984.

[4] K. Atanassov, Intuitionistic fuzzy sets, Fuzzy Sets and Systems, Vol. 20, no. 1, pp. 87-96, 1996.

[5] K. Atanassov, More on intuitionistic fuzzy sets, Fuzzy Sets and Systems, Vol. 33, no. 1, pp. 37-46, 1989.

[6] L. A. Zadeh, Outline of a new approach to analysis of complex systems and decision processes, IEEE Trans. on Systems, Man and Cybernet., Vol. 3, pp. 28-44, 1973.

[7] E. Barrenechea, H. Bustince, B. De Baets, C. Lopez-Molina, Construction of Interval-Valued Fuzzy Relations With Application to the Generation of Fuzzy Edge Images, IEEE Trans. on Fuzzy Systems, Vol.19, no.5, pp.819830, Oct. 2011.

[8] H. Bustince, Interval-valued Fuzzy Sets in Soft Computing, International Journal of Computational Intelligence Systems, Vol. 3, no. 2, pp. 215-222, 2010 .

[9] H. Bustince, E. Barrenechea, M. Pagola, J. Fernandez, C. Guerra, P. Couto, and P. Melo-Pinto, Generalized Atanassov's intuitionistic fuzzy index: Construction of Atanassov's fuzzy entropy from fuzzy implication operators, International Journal of Uncertainty, Fuzziness and Knowledge-Based Systems, Vol. 19, 1, pp. 51-69, 2011. 
[10] H. Bustince, M. Pagola, E. Barrenechea, J. Fernandez, P. Melo-Pinto, P. Couto, H.R. Tizhoosh, J. Montero, Ignorance functions. An application to the calculation of the threshold in prostate ultrasound images, Fuzzy Sets and Systems, Vol. 161, 1, pp. 20-36, 2010.

[11] W. L. Gau, D. J. Buehrer, Vague Sets, IEEE Trans. on Systems Man Cybernet, 23 (2), 610-614, 1993.

[12] F. Smarandache, A unifying field in logics: neutrosophic logic, MultipleValued Logic, Vol. 8, pp. 385-438, 2002.

[13] R. R. Yager, On ordered weighted averaging aggregation operators in multi-criteria mecision making, IEEE Transactions on Systems, Man and Cybernetics, Vol. 18, pp. 183-190, 1998.

[14] R. R. Yager, and D. P. Filev, Induced ordered weighted averaging operators, IEEE Trans Syst Man Cybernetics B, Vol. 29, pp. 141-150, 1999.

[15] R. R. Yager, The induced fuzzy integral aggregation operator, International Journal of Intelligent Systems, Vol. 17, pp. 1049-1065, 2002.

[16] T. Calvo, G. Mayor, and R. Mesiar (Eds.), Aggregation operators: new trends and applications, Studies in Fuzziness and Soft Computing, Vol. 97, Physica-Verlag, Heidelberg, 2002, pp. 224-244.

[17] F. Smarandache, Definition of neutrosophic logic. A generalization of the intuitionistic fuzzy logic, Proc. 3rd Conf. Eur. Soc. Fuzzy Logic Tech. (EUSFLAT, 2003), pp. 141-146, 2003.

[18] F. Smarandache, Neutrosophic set. A generalization of the intuitionistic fuzzy set, Intern. J. Pure Appl. Math., Vol. 24, pp. 287-97, 2005.

[19] M. Hanmandlu and A. Das, 2011. Content-based Image Retrieval by Information Theoretic Measure. Defence Science Journal, Vol. 61, no. 5, pp. $415-430$

[20] M. Aggarwal, M. Hanmandlu, Representing uncertainty with information sets, IEEE Transactions on Fuzzy Systems, Vol. 24, no. 1, pp. 1-15, Feb. 2016.

[21] M. Aggarwal, Hesitant Information Sets and Applications in Group Decision Making, Applied Soft Computing, 2018. 
[22] V. Torra, Hesitant Fuzzy Sets, International Journal of Intelligent Systems, Vol. 25, no. 6, pp. 529-539, 2010.

[23] M. Xia, Z. Xu, Hesitant fuzzy information aggregation in decision making, International Journal of Approximate Reasoning, Vol. 52, no. 3, pp. 395 - 407, 2011.

[24] M. Mizumoto, K. Tanaka, Some properties of fuzzy sets of type 2, Inform Control, 1976, Vol. 31, $312-340$.

[25] D. Dubois, H. Prade, Fuzzy sets systems, New York: Academic Press, 1980.

[26] Z. Zhang, W. Yu, L. Martínez and Y. Gao, Managing Multigranular Unbalanced Hesitant Fuzzy Linguistic Information in Multiattribute Large-Scale Group Decision Making: A Linguistic Distribution-Based Approach, IEEE Transactions on Fuzzy Systems, vol. 28, no. 11, pp. 2875-2889, Nov. 2020.

[27] Z. Zhang, J. Gao, Y. Gao, W. Yu, Two-sided matching decision making with multi-granular hesitant fuzzy linguistic term sets and incomplete criteria weight information, Expert Systems with Applications, Vol. 168, 2021, 114311.

[28] CC. Li, Y. Gao, Y. Dong, Managing Ignorance Elements and Personalized Individual Semantics Under Incomplete Linguistic Distribution Context in Group Decision Making. Group Decision and Negotiation, 30, 97-118, 2021

[29] F. Meng, S-M Chen, S. Zhang, Group decision making based on acceptable consistency analysis of interval linguistic hesitant fuzzy preference relations, Information Sciences, Vol. 530, pp. 66-84, 2020

[30] P. Ren, Z. Xu, X. Wang, X-J Zeng, Group decision making with hesitant fuzzy linguistic preference relations based on modified extent measurement, Expert Systems with Applications, Vol. 171, 2021, 114235,

[31] W. Liang, M. Goh, Y-M Wang, Multi-attribute group decision making method based on prospect theory under hesitant probabilistic fuzzy environment, Computers \& Industrial Engineering, Vol. 149, 2020, 106804 
[32] W. Zeng, Y. Xi, Q. Yin, P. Guo, Weighted dual hesitant fuzzy set and its application in group decision making, Neurocomputing, 2020,

[33] J. Liu, C. Huang, J. Song, P. Du, F. Jin, H. Chen, Group decision making based on the modified probability calculation method and DEA crossefficiency with probabilistic hesitant fuzzy preference relations, Computers \& Industrial Engineering, Vol. 156, 2021, 107262,

[34] P. Liu, X. Zhang, W. Pedrycz, A consensus model for hesitant fuzzy linguistic group decision-making in the framework of Dempster-Shafer evidence theory, Knowledge-Based Systems, Vol. 212, 2021, 106559,

[35] Y. Zheng, Z. Xu, Y. He, Y. Tian, A hesitant fuzzy linguistic bi-objective clustering method for large-scale group decision-making, Expert Systems with Applications, Vol. 168, 2021, 114355,

[36] N. Wu, Y. Xu, X. Liu, H. Wang, E. Herrera-Viedma, Water-EnergyFood nexus evaluation with a social network group decision making approach based on hesitant fuzzy preference relations, Applied Soft Computing, Vol. 93, 2020, 106363,

[37] M. Yazdani, A. Mohammed, C. Bai, A. Labib, A novel hesitant-fuzzybased group decision approach for outsourcing risk, Expert Systems with Applications, Vol. 184, 2021, 115517

[38] R. M. Rodriguez, L. Martinez and F. Herrera, Hesitant Fuzzy Linguistic Term Sets for Decision Making,IEEE Transactions on Fuzzy Systems, vol. 20, no. 1, pp. 109-119, Feb. 2012.

[39] H. Wang, Extended hesitant fuzzy linguistic term sets and their aggregation in group decision making, International Journal of Computational Intelligence Systems, 8 (1), pp. 14-33, 2015 